\title{
Ricerca di HPV-DNA e tipizzazione virale nella diagnostica di prevenzione del carcinoma della cervice uterina
}

\author{
Mauro Carcheri, Grazia Lacitignola, Renato Riva, Roberto Capuzzo, Mentasti Massimo, \\ Ventura Agostina, Ragusa Tommaso', Cirillo Rossana ${ }^{2}$
}

Azienda Ospedaliera "Villa Scassi”, Dipartimento di Patologia Clinica - Laboratorio Analisi Chimico-Cliniche e Microbiologiche

' Dipartimento di Patologia Clinica - U.O.Anatomia Patologica

${ }^{2}$ U.O. Ostetricia e Ginecologia

\section{Detection and typing of HPV-DNA in cervical cancer screening}

Key Words: Human papillomavirus, HPV, Proliferative lesions, Cervical cancer

\section{SUMMARY}

Papillomaviruses are relatively ubiquitous and have been described as causative agents for epithelial lesions in a wide variety of animals as well as in humans. Approximately 30 HPV types have been isolated from anogenital epithelium (cervix, vagina, vulva, rectum and penis).

HPVs induce a variety of proliferative lesions, but only the "high-risk" types are associated with anogenital cancers. "Low-risk" viral types include HPV-6 and HPV-II; "high-risk" types include HPV-I6, I8, 3I and 56. HPVs "high-risk" types are more strongly associated with high-grade lesions (HSIL) than they are with low-grade lesions (LSIL).

The traditional process for cervical cancer screening programs (PAP test) is vulnerable to air drying artifacts and has limits to sensitivity, since as many as 90 percent of collectet cells can be discarted with collection device.

The present study was undertaken to assess the screening performance of HPV-DNA typing in a sample of I42 women drawn from a routine screening for the prevention of cervical cancer.

The results indicate that HPV-DNA screening and typing, used together with PAP test, can improve the detection of patients with cervical disease and can serve as a quality assurance indicator in cervical cancer screening programs.

\section{INTRODUZIONE}

I Papillomavirus sono piccoli virus a simmetria icosaedrica, privi di pericapside, con genoma costituito da DNA a doppia elica ( 7.900 coppie di basi), riconosciuti come agenti eziologici di processi proliferativi nei tessuti epiteliali squamosi. Possiedono uno specifico tropismo per gli epiteli pluristratificati dove si replicano, dando luogo a caratteristiche lesioni che coincidono con la presenza di coilociti, cellule con grossi vacuoli perinucleari e addensamento di citoplasma.

L'interazione HPV-cellula realizza quadri diversi in funzione del differente stato replicativo virale: infezione allo stato latente in cui il genoma virale va incontro ad una replicazione in forma episomale, infezione produttiva in cui attraverso la propria replicazione il virus induce la proliferazione dell'epitelio squamoso, integrazione del DNA virale in quello cellulare portando ad una maggiore probabilità di sviluppo di lesioni trasformanti. Un importante fattore di progressione neoplastica sembra essere la capacità del virus di determinare un'infezione persistente senza apparenti manifestazioni cliniche, ma con un'espressione protratta dei geni virali (8).

La proliferazione epiteliale o fibroepiteliale della cute e delle mucose normalmente presenta caratteristiche di benignità e regredisce spontaneamente, ma in un certo numero di casi, solitamente correlati a specifici tipi virali, può invece evolvere in situazioni spiccatamente neoplastiche.

I Papillomavirus che infettano l'uomo (HPV) sono circa 80 tipi e di questi almeno 30 infettano l'apparato genitale femminile e maschile rendendo l'infezione da HPV la patologia a trasmissione sessuale più diffusa nel mondo (2).

Secondo l'inquadramento clinico tradizionale gli HPV genitali vengono suddivisi in tipi a "basso rischio", "medio rischio" ed "alto rischio" oncogeno.

I tipi a basso rischio (prevalentemente HPV-6 e HPV-11) sono riconosciuti come gli agenti causali dei condilomi acuminati, lesioni vegetative di tipo benigno.

I tipi a medio rischio (HPV-33, 35, 52, 56...) sono talvolta associati a lesioni neoplastiche.

I tipi ad alto rischio (HPV-16, 18, 31, 45) sono stati identificati in più dell' $80 \%$ dei carcinomi 
cervicali $(1,8)$.

Le manifestazioni cliniche e citologiche derivate da un'infezione da HPV (Squamous Intraepitelial Lesion, SIL) sono di norma limitate nel loro sviluppo dall'azione del sistema immunitario del soggetto colpito (tramite la produzione di linfociti $\mathrm{T}$ citotossici che riconoscono e distruggono le cellule infette).

Le SIL sono suddivise in LSIL (lesioni di basso grado) e HSIL (lesioni di alto grado); solo il 15\% delle LSIL evolve in HSIL anche perché la maggior parte delle LSIL regredisce in maniera spontanea $(4,6)$.

L'infezione da HPV è diffusa nell' $80 \%$ della popolazione sessualmente attiva, con un picco nei soggetti con età tra i 22-25 anni.

Come le infezioni da Herpes, anche le infezioni da HPV possono presentare episodi di ricorrenza delle lesioni, intervallate da periodi di assenza di segni clinici.

L'intervallo di tempo tra l'infezione iniziale e la comparsa del carcinoma è spesso molto lungo e, comunque, non tutti i soggetti con un'infezione da HPV ad "alto rischio" sviluppano un tumore.

Sono state dimostrate, con una buona frequenza, infezioni multiple da HPV nello stesso soggetto.

La prevenzione del carcinoma della cervice uterina si basa, dagli anni '60, sull'utilizzo del PAP test quale test di screening citologico.

Il PAP test ha, comunque, una sensibilità di circa il 90\%, attualmente al Pap Test ed all'eventuale successivo esame colposcopico, devono essere affiancate, in particolare nei casi di dubbia interpretazione citologica (presenza di ASC - US ASC-H) o nei casi di positività (presenza di LSIL o HSIL) metodiche di biologia molecolare in grado di mettere in evidenza con elevata sensibilità la presenza di HPV nelle cellule in esame. Inoltre può essere eseguita una tipizzazione dei ceppi virali ad elevato potenziale oncogeno $(3,5$, 9).

Nello studio che segue presentiamo i risultati di indagini di biologia molecolare riguardanti l'infezione da HPV eseguite nel periodo di un anno circa (dal 16 Gennaio 2002 ad oggi) in una popolazione di 142 donne sottopostasi ad accertamenti preventivi per il carcinoma cervicale.

\section{MATERIALI E METODI}

Sono state prese in esame 142 donne che si sono presentate presso i nostri Ambulatori per indagini di prevenzione del carcinoma della cervice (colposcopia, biopsia o PAP test).

Le pazienti sono state suddivise in quattro fasce d'età:

$$
\text { > Fascia 1: da } 25 \text { a } 30 \text { anni } 30 \text { pazienti }
$$

$>$ Fascia 2: da 30 a 35 anni

52 pazienti

40 pazienti

20 pazienti

Il materiale da sottoporre ad esame è costituito da un cyto-brush endocervicale, eseguito con un apposito tampone che, dopo il prelievo, viene inserito in una apposita provetta sterile contenente $0.5 \mathrm{ml}$ di soluzione di trasporto (una miscela di penicillina a $10.000 \mathrm{U} / \mathrm{ml}$, streptomicina a 10 $\mathrm{mg} / \mathrm{ml}$ e anfotericina a $25 \mu \mathrm{g} / \mathrm{ml}, 5 \mathrm{ml}$ di soluzione in $500 \mathrm{ml}$ di fisiologica sterile).

I campioni prelevati vengono conservati a $-20^{\circ} \mathrm{C}$ sino al momento dell'esecuzione dell'esame (di media alcuni giorni dopo il prelievo).

Al momento dell'esecuzione del test, dopo scongelamento, le provette contenenti i cyto-brush sono vigorosamente vortexate per 15 ", quindi si trasferisce il campione in provette Eppendorf cercando di recuperare tutto il materiale presente nel tampone. Le provette sono sottoposte a centrifugazione a 12.000 g per 5', quindi si procede alla lisi cellulare e all'estrazione del DNA utilizzando un kit commerciale per la rivelazione e tipizzazione dei vari tipi di Papillomavirus umano a basso ed alto rischio di trasformazione neoplastica (AmpliSens HPV - Nuclear Laser Medicine).

Il DNA estratto è stato sottoposto ad amplificazione utilizzando $4 \mathrm{mix}$ di primers diversi mediante una Multiplex-PCR che amplifica simultaneamente in una provetta un gruppo di target virali ed un Controllo Interno (IC).

DNA bersaglio dell'IC è la regione del genoma umano codificante il gene $\beta$-globinico e quindi deve essere presente in tutti i campioni.

Le mix di primers sono:

$>$ mix a "basso rischio"

primers per i tipi virali 6 e 11

$>$ mix 16-35

primers per i tipi virali $16,31,33,35$

$>\operatorname{mix} 18-59$

primers per i tipi virali $18,39,45,59$

$>\operatorname{mix} 52-66$

primers per i tipi virali 52, 56, 58, 66

La rivelazione della presenza di amplificato specifico viene eseguita mediante elettroforesi su gel di agarosio al $2 \%$ contenente $0.5 \mu \mathrm{g} / \mathrm{ml}$ di etidio bromuro. Il gel è stato fatto correre a 10 Volts $/ \mathrm{cm}$ per circa 2 ore.

Per evidenziare le bande corrispondenti ai diversi tipi virali è stata utilizzata un marcatore di pesi molecolari (50bp DNA Step Ladder) che evidenzia bande da 50 a 800 bp.

A fine corsa il gel è stato posto su transilluminatore a UV (303 nm) e fotografato con filtro UV e pellicola Polaroid. 
I campioni che non presentano la banda dell' IC non sono ritenuti idonei.

\section{RISULTATI E DISCUSSIONE}

Delle 142 donne prese in esame 6 presentavano un'infezione da HPV a "basso rischio" (4.2\%) e 30 un'infezione da tipi virali a "medio o alto rischio" $(21.12 \%)$ (percentuale delle infezioni da basso rischio+medio/alto rischio sul totale del campione esaminato $=25.3 \%$ )

La fascia 1 ha presentato 1 caso di infezione da HPV a "basso rischio" e 8 casi di infezione da tipi virali a "medio o alto rischio" (rispettivamente $0.7 \%$ e $5.6 \%$ sul totale delle pazienti esaminate $3.3 \%$ e $26.6 \%$ sulla fascia d'età).

La fascia 2 ha presentato 4 casi di infezione da HPV a "basso rischio" e 13 casi di infezione da tipi virali a "medio o alto rischio" (rispettivamente $2.8 \%$ e $9.15 \%$ sul totale delle pazienti esaminate $-7.7 \%$ e $25.0 \%$ sulla fascia d'età).

La fascia 3 ha presentato 1 caso di infezione da HPV a "basso rischio" e 6 casi di infezione da tipi virali a "medio o alto rischio" (rispettivamente $0.7 \%$ e $4.2 \%$ sul totale delle pazienti esaminate $2.5 \%$ e $15.0 \%$ sulla fascia d'età).

La fascia 4 ha presentato 0 casi di infezione da HPV a "basso rischio" e 3 casi di infezione da tipi virali a "medio o alto rischio" (rispettivamente $0 \%$ e $2.1 \%$ sul totale delle pazienti esaminate $0 \%$ e $15 \%$ sulla fascia d'età) tabella 1

I risultati ottenuti confermano la relativamente elevata percentuale di diffusione degli HPV nella popolazione sessualmente attiva $(25.3 \%)$ e confermano che un'elevata percentuale $(21.12 \%)$ delle infezioni è sostenuta da tipi virali ad alto rischio oncogeno.

Le fasce d'età più giovani presentano le percentuali di infezione più elevate $(30.0 \%$ per la fascia da 25 a 30 anni, $32.7 \%$ per quella da 30 a 35 ).

Ci sembra importante sottolineare che, in tutte le fasce d'età, e soprattutto nelle prime due, il maggior numero di infezioni è sostenuto da tipi virali a medio/alto rischio oncogeno (particolarmente alte sono le percentuali d'infezione dei tipi virali HPV-16 e HPV-18 per i tipi ad alto rischio e di HPV-56 per quelli a medio rischio).

La diffusione dell'infezione da HPV e le elevate percentuali a carico dei tipi ad alto rischio indicano, pertanto, nella tipizzazione di HPV dopo amplificazione mediante PCR un test da affiancare in maniera decisa alla metodiche tradizionali (PAP test, ecc) nella prevenzione del carcinoma della cervice uterina.

I tipi virali identificati sono stati:

\begin{tabular}{lcc}
\hline TIPO VIRALE & NUMERO & \% SUL TOTALE \\
\hline HPV-6 & 5 & 3.5 \\
\hline HPV-II & 1 & 0.7 \\
\hline HPV-16 & 10 & 7.0 \\
\hline HPV-18 & 6 & 4.2 \\
\hline HPV-3I & 3 & 2.1 \\
\hline HPV-56 & 11 & 7.7 \\
\hline
\end{tabular}

Nelle fasce d'età prese in considerazione le percentuali d'identificazione dei vari tipi di HPV sono state:

\begin{tabular}{lllllll}
\hline FASCIA D'ETA & HPV-6 & HPV-II & HPV-I6 & HPV-I8 & HPV-3I & HPV-56 \\
\hline 1 & 3.3 & 0.0 & 6.6 & 10.0 & 3.3 & 6.6 \\
\hline 2 & 5.7 & 1.9 & 7.6 & 1.9 & 3.8 & 11.5 \\
\hline 3 & 2.5 & 0.0 & 7.5 & 2.5 & 0.0 & 5.0 \\
\hline 4 & 0.0 & 0.0 & 0.0 & 5.0 & 0.0 & 5.0 \\
\hline
\end{tabular}

In totale sono stati riscontrati 5 casi di infezione doppia o multipla:

fascia I: 2 casi (HPV-16 e HPV-31 - HPV-18 e HPV-56)

fascia 2: 2 casi (HPV-16 e HPV-56 - HPV-18 e HPV-56)

fascia 3: nessun caso

fascia 4: I caso (HPVI6, HPV-I8 e HPV-56)

Tabella I. Tipizzazione di HPV a "basso rischio", a "medio" ed a "alto rischio: numero di virus identificati e distribuzione percentuale sul totale dei campioni esaminati e per fascia d'età

\begin{tabular}{|c|c|c|c|c|c|c|c|c|}
\hline \multirow{3}{*}{$\begin{array}{l}\text { FASCIA } \\
\text { ETȦ }\end{array}$} & \multirow{3}{*}{$\begin{array}{c}N . \\
\text { CAMPIONI }\end{array}$} & \multicolumn{3}{|c|}{ LR } & \multicolumn{3}{|c|}{ HR } & \multirow{3}{*}{$\begin{array}{l}\text { TOT \% PER } \\
\text { FASCIA ETÄ }\end{array}$} \\
\hline & & N. VIRUS & TOT & FASCIA & N. VIRUS & TOT & $\overline{\text { FASCIA }}$ & \\
\hline & & ID & $\%$ & $\%$ & ID & $\%$ & $\%$ & \\
\hline $25-30$ & 30 & 1 & 0.7 & 3.3 & 8 & 5.6 & 26.6 & 30.0 \\
\hline $31-35$ & 52 & 4 & 2.8 & 7.7 & 13 & 9.2 & 25 & 32.7 \\
\hline $36-40$ & 40 & $I$ & 0.7 & 2.5 & 6 & 4.2 & 15 & 17.5 \\
\hline$>40$ & 20 & 0 & 0.0 & 0.0 & 3 & 2.1 & 15 & 15.0 \\
\hline
\end{tabular}

Tabella 2. Tipi di HPV identificati: distribuzione percentuale sul totale dei campioni esaminati e per fascia d'età

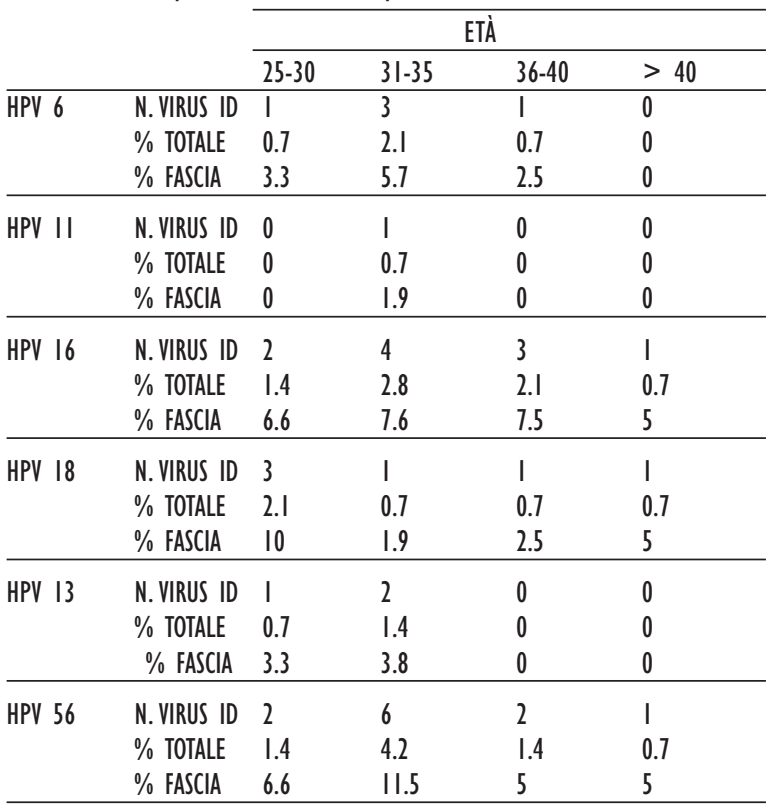




\section{BIBLIOGRAFIA}

1. Bosch FX, Manos MM, Mu-oz N, Sherman M et al. Prevalence of Human Papillomavirus in Cervical Cancer: a Worldwide Perspective. International Biological Study on Cervical Cancer (IBSCC) Study Group. 1995. J Natl Cancer Inst. 87:796-802

2. De Villiers EM, 1994. Human pathogenic papillomavirus types : an update. Curr.Top.Microbiol.Immunol. 186:1-12

3. Ferris DG, Wright TC, Litaker MS, Richart RM et al. Comparison of two tests for detecting carcinogenic HPV in women with Papanicolau smear reports of ASCUS and LSIL. Journal of Family Practice 1998; 46(2): 136-141

4. Holly EA. Cervical intraepithelial neoplasia, cervical cancer and HPV. Annu Rev Public Healt 1996; 17:69-84

5. Manos MM, Kinney WK, Hurley LB et al. Identifying women with cervical neoplasia: using human papillomavirus DNA testing for equivocal Papanicolau results. JAMA 1999; 281(17): 16051610

6. Nobbenhuis MA, Walboomers JM, Helmerhorst TJ et al. Relation of human papillomavirus status to cervical lesions and conseguences for cervical-cancer screening: a prospective study. Lancet 1999; 354(9172): 20-25

7. Park TW, Fujiwara H, Wright TC. Molecular biology of cervical cancer and its precursors. Cancer 1995 Nov 15;76(10 Suppl):1902-13

8. Voudsen K. 1993. Interaction of human papillomavirus transforming proteins with the products of tumor suppressor genes. FASEB J. 7:872-879

9. Wright TC jr, Denny L, Khun L et al. HPV DNA testing of self-collected vaginal samples compared with cytologic screening to detect cervical cancer. JAMA 2000; 283(1): 81-6.

Mauro Carcheri

Azienda Ospedaliera "Villa Scassi", Laboratorio Analisi, Settore Virologia e Biologia Molecolare

C.so O.Scassi 1; 16149 Genova Tel. 0104102 393;

e-mail: virologia@villascassi.it 